\title{
Zinc Status and Febrile Seizures: Results from a Cross-sectional Study
}

\author{
Janani Arul ${ }^{1}$ Peter Prasanth Kumar Kommu ${ }^{1} \quad$ Ananthanarayanan Kasinathan ${ }^{1} \quad$ Lopamudhra Ray ${ }^{2}$ \\ Lalitha Krishnan ${ }^{1}$
}

1Department of Pediatrics, Pondicherry Institute of Medical

Sciences, Puducherry, India

2Department of Biochemistry, Pondicherry Institute of Medical

Sciences, Puducherry, India

\begin{abstract}
Address for correspondence Ananthanarayanan Kasinathan, MD, DM, Department of Pediatrics, Pondicherry Institute of Medical Sciences, Puducherry 605014, India

(e-mail: cerebratelife@gmail.com).
\end{abstract}

J Neurosci Rural Pract 2020;11:597-600

\begin{abstract}
Objective To estimate the serum zinc levels in children under the age of 5 years with febrile seizures and febrile children without seizures

Materials and Methods In this cross-sectional study from 2017 to 2018, 40 children with febrile seizures (simple and complex) were taken as cases. Forty age- and sexmatched febrile children without convulsions were recruited as controls. Serum zinc estimates were analyzed using a spectrophotometer (Biolis 50i-Autoanalyzer).

Statistical Analysis The demographic variables and serum zinc estimates were analyzed using the Mann-Whitney test. The odds ratio was used to calculate the association of zinc deficiency in febrile seizures; $5 \%$ level of significance was considered.

Keywords

- Zinc

- Febrile seizures

- Epilepsy

- Children Results The mean serum concentrations of zinc in the cases and controls were $83.8 \pm 33.1 \mu \mathrm{g} / \mathrm{dL}$ and $116.3 \pm 30.3 \mu \mathrm{g} / \mathrm{dL}$, respectively $(p=0.002)$. Hypozincemia defined by "a serum zinc level of less than $63 \mu \mathrm{g} / \mathrm{dL}$ " was found in $12(30 \%)$ cases and $2(5 \%)$ controls with an odds ratio of $8: 1$.

Conclusion Children with febrile seizures had significantly reduced concentrations of zinc in the serum.
\end{abstract}

\section{Introduction}

Febrile seizures are a commonly encountered neurological source of hospital attendance in children with a worldwide incidence of 2 to $8 \%{ }^{1}$ The peak age for the occurrence is 18 to 24 months, and the majority (90\%) of the children report the first episode before the age of 3 years. ${ }^{2}$ The operational definition proposed by "International League Against Epilepsy," "a seizure occurring in childhood after one month of age, associated with a febrile illness not caused by an infection of the central nervous system, without previous neonatal seizures or a previous unprovoked seizure, and not meeting criteria for other acute symptomatic seizures" emphasizes the judicious use of select laboratory testing to rule out intracranial infection before making a diagnosis of febrile seizures. ${ }^{3}$ The age-specific predilection of febrile seizures is still unclear, and a complex interplay of genetic and environmental factors have been postulated. A robust relationship with febrile seizures and genetic epilepsy (voltage-gated sodium channelopathy) has been established in one-third of children with positive family history. ${ }^{4}$ However, a significant pedigree chart is absent in several children with febrile seizures and routine genetic testing is not warranted. Additional factors considered in the pathogenesis include dyselectrolytemia (hyponatremia), pyridoxine deficiency, human herpes virus 6 , and disorders of trace elements, primarily zinc, iron, and copper. ${ }^{5}$ Among them, zinc status in children with febrile seizures has drawn substantial attention, given its high-concentration in the brain and potential role in synaptic neurotransmission. This has been complimented by animal studies, especially mouse models. Zinc regulates the rate-limiting enzyme in GABA synthesis and also prevents excitatory neuronal discharge by facilitating the inhibitory effect of NMDA receptors. ${ }^{6}$ This largely inhibitory outcome is diminished following hypozincemia secondary to
License terms

10.1055/s-0040-1715992

ISSN 0976-3147. 
endogenous pyrogens released during the febrile illness. Data from prospective studies on zinc status in febrile seizures do not run in parallel with conflicting results. Given the limited information from the Indian subcontinent, this study was designed to ascertain the relationship between hypozincemia and febrile seizures in children.

\section{Materials and Methods}

This prospective cross-sectional study was held at a tertiary teaching institute in Puducherry, India, over 18 months from January 2017 to June 2018 after obtaining approval from the Institutional Ethics Committee (Ref. No IEC:RC/15/34). Children aged6months to five years, who presented to the pediatric outpatient department, satisfying the study definition of febrile seizures were taken as cases, such aschildren on zinc therapy for diarrhea, pneumonia, acrodermatitis enteropathica, and severely malnourished children; children with dyselectrolytemia; children with clinical signs of intracranial infection were excluded from the study. An equal number of age- and sex-matched febrile children without seizures were taken as controls. Demographic details including birth history, family history, and details about the febrile seizures (age of onset, type of seizure, duration, treatment, and prophylactic therapy) were collected. Parental consent was obtained prior to enrolment. Furthermore, infants underwent cerebrospinal spinal fluid (CSF) analysis to rule out intracranial infections.

Four milliliters of blood samples were drawn within 24 hours of admission. Two milliliters of the samples were centrifuged, and the separated serum was analyzed for zinc levels using a spectrophotometer (Biolis50i- Autoanalyzer) and $560 \mathrm{~nm}$ wavelength with the help of aFAR diagnostic kit (zinc reagent $=5$ bromo, 2 pyridylazo-5N-propylN-sulphonopropyl phenol). A serum zinc level of less than $65 \mathrm{mcg} / \mathrm{mL}$ was taken as hypozincemia. Also, serum levels of electrolytes (sodium, potassium, calcium and magnesium), C-reactive protein, and albumin levels were analyzed to estimate the bound form of zinc. All children were followed-up until the time of discharge from the hospital.

\section{Data Presentation and Statistical Analysis}

The incidence of febrile seizure as per literature was found to be 2 to $5 \%$. With an error of $5 \%$ and a confidence interval of $95 \%$, a sample size of 80 children was estimated. The results were recorded on a proforma that was then analyzed using SPSS 20.2 software. The demographic variables were analyzed, and statistical significance was estimated using the Mann-Whitney test. The odds ratio was used to calculate the association of zinc deficiency in febrile seizures. The box and whisker plot was used to analyze statistical significance of serum zinc levels; $5 \%$ level of significance was considered.

\section{Results}

From January 2017 to June 2018, among the 12330 children presenting to the pediatric outpatient/accident and emergency services, 6480 children with fever were screened for eligibility; 40 children with febrile seizures as cases and 40 febrile children without convulsions as controls were recruited for the study. - Table 1 depicts the profile of children recruited in the study. The mean age (standard deviation [SD]) of the cases was 17 months and children aged 12 to 36 months accounted for $55 \%$ of all included cases. A slight female preponderance was noted in both groups. Amongst the cases, 30 children (77\%) presented with simple febrile seizures and 10 had complex febrile seizures. One-fifth of the cases had a family history of febrile seizures. Twenty-eightcases (70\%) sought medical attention for the first episode of febrile seizures, and clonic seizures was the common semiology in $63 \%$ of the cases. Pneumonia (40\%), viral upper respiratory tract infection (25\%), scrub typhus (12\%), and urinary tract infection (11\%) were the etiology of fever in the control group.

The mean serum concentrations of zinc in the cases and controls were $83.8 \pm 33.1 \mu \mathrm{g} / \mathrm{dL}$ and $116.3 \pm 30.3 \mu \mathrm{g} / \mathrm{dL}$, respectively. The mean serum zinc status in the febrile seizure group were significantly different $(p=0.002)$ from the controls ( - Fig. 1). Hypozincemia, defined by "a serum zinc concentration of less than $63 \mu \mathrm{g} / \mathrm{dL}$ " was found in $12(30 \%)$ cases

Table 1 Clinical characteristics of children with febrile seizures and febrile children without seizures

\begin{tabular}{|c|c|c|}
\hline Variable & $\begin{array}{l}\text { Cases } \\
(n=40)(\%)\end{array}$ & $\begin{array}{l}\text { Controls } \\
(n=40)(\%)\end{array}$ \\
\hline Females & $22(55 \%)$ & 19 (47.5\%) \\
\hline \multicolumn{3}{|l|}{ Age, in months } \\
\hline$<12$ & $7(17.5 \%)$ & $12(40 \%)$ \\
\hline $13-36$ & $22(55 \%)$ & $23(57.5 \%)$ \\
\hline $37-60$ & $11(27.5 \%)$ & $5(12.5 \%)$ \\
\hline \multicolumn{3}{|l|}{ Fever duration, in hours } \\
\hline$<24$ & $30(75 \%)$ & $3(7.5 \%)$ \\
\hline$>24$ & $10(25 \%)$ & 37 (92.5\%) \\
\hline Mean peak temperature $\left({ }^{\circ} \mathrm{C}\right)$ & $38.4 \pm 0.9$ & $38.1 \pm 0.6$ \\
\hline \multicolumn{3}{|l|}{ Etiology } \\
\hline $\begin{array}{l}\text { Viral Upper respiratory tract } \\
\text { infection }\end{array}$ & $29(72 \%)$ & $10(25 \%)$ \\
\hline Pneumonia & $5(12 \%)$ & $16(40 \%)$ \\
\hline Others & $6(16 \%)$ & $14(35 \%)$ \\
\hline Mean serum CRP ( \pm SD mg/L) & $13.1 \pm 4.4$ & $11.8 \pm 3.7$ \\
\hline Mean serum albumin $( \pm \mathrm{SD} \mathrm{g} / \mathrm{dL})$ & $4.6 \pm 1.2$ & $4.3 \pm 1.9$ \\
\hline Mean serum zinc $\left( \pm S D\right.$ mcg/dL) ${ }^{a}$ & $83.8 \pm 33.1$ & $116.3 \pm 30.3$ \\
\hline
\end{tabular}

Note: Data presented as $n$ (\%) unless otherwise stated.

${ }^{a}$ Comparison was statistically significant with $p$-value of 0.002 .

Other variables were not statistically significant. 


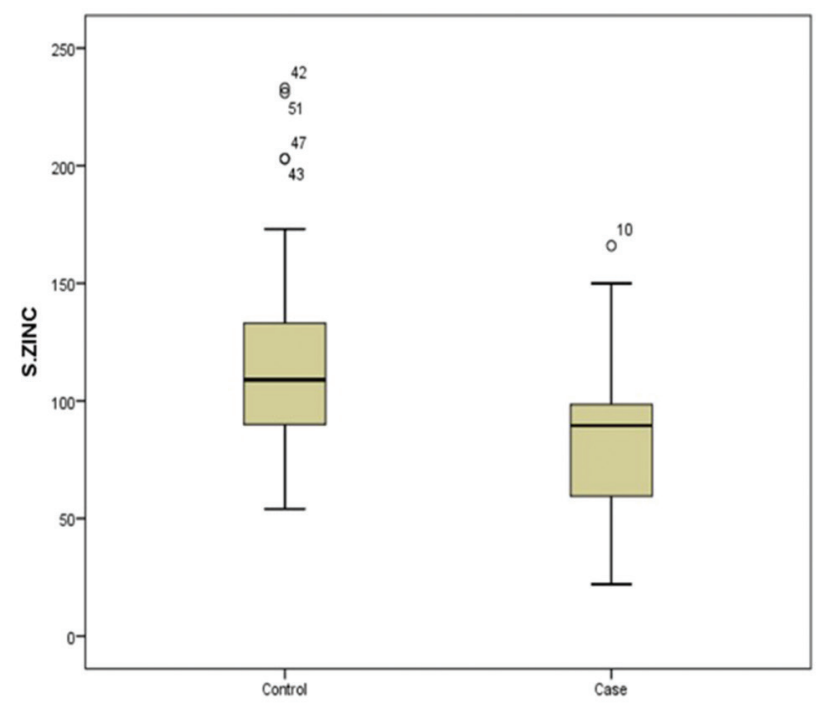

Fig. 1 Comparison of serum zinc concentration in $\mathrm{mcg} / \mathrm{dL}$ for cases and controls by box and whisker plot.

and $2(5 \%)$ controls with an odds ratio of 8.1. Furthermore, age, sex, and degree of fever had no significant association with serum zinc levels

\section{Discussion}

The current study showed a significantly lower serum concentration of zinc in children with febrile seizures when compared with age- and sex-matched controls of febrile children with no seizures. The results of the study are congruent with the largest study from Nigeria by Okposio et al who assessed zinc status in 180 children. ${ }^{7}$ The mean serum zinc estimates of the febrile seizure group $(83.8 \mu \mathrm{g} / \mathrm{dL})$ was higher than the study from Nigeria. The variation in the serum zinc levels may be attributed to the epidemiological difference in basal zinc and nutritional status of the cohort. A humble attempt at a systematic review by Nasehi et al yielded a temporal association of relatively low zinc levels in children with febrile seizures in comparison to fever-matched controls with no seizures. ${ }^{8}$ However, there are conflicting reports from Celik et al and Cho et al who did not find the difference remarkable. ${ }^{9}$ Weak sample size and technical differences in serum zinc estimation were the reasons ascribed for the poor association. Nonetheless, a critical analysis of the published data provides very little information on the proportion of hypozincemia for risk stratification. In the present study, hypozincemia was noted in $30 \%$ of the children with febrile seizures. This is proportionately low when compared with a recent study of 50children with febrile seizures by Hameed et al in Egypt where hypozincemia was obtained in one half of the cohort. ${ }^{10}$ The true incidence of hypozincemia in children with febrile seizures is arguable, as a parallel study from Iran did not identify a solitary case of hypozincemia. ${ }^{11}$ Rigid criteria of less than 40 for zinc deficiency may be the reason for this dissimilarity in the study.
In addition, lower serum concentrations of zinc are not sine qua non with febrile seizures and has also been evaluated in patients with well-controlled and difficult to control epilepsy. A preliminary study by Wojciak et al resonated with the observation of lower serum zinc levels in children with epilepsy as well. ${ }^{12}$ Although it is unclear what the ultimate meaning of these findings portend, it extends the scope for further research into the role of zinc in synaptic neurotransmission and its pathogenesis in seizures. The possible explanation for the low serum zinc status in febrile seizures includes redistribution of zinc into the liver and infection-induced hypozincemia. Zinc is ubiquitous within cells and has predefined catalytic, structural, and regulatory functions. During active inflammation secondary to stressors such as fever or seizures, cytokines, which are released as an acute phase response, activate the hepatic synthesis of metallothionein. Metallothionein is an intracellular metal-binding protein whichincreases the hepatic absorption of zinc with a consequent drop in serum zinc levels. ${ }^{13}$ Elevated C-Reactive protein as a marker for acute phase response has been considered in the interpretation of serum zinc concentrates by Lee et al. ${ }^{14}$ Serum zinc and serum CRP estimates in preschoolers with febrile and afebrile convulsions were analyzed and CRPadjusted serum zinc concentration was remarkably reduced in the febrile seizures group. Retrospective analysis of the samples was one of the drawbacks of the study. The current study did not show any difference in serum zinc levels with age, gender, peak temperature, serum CRP, and serum albu$\mathrm{min}$. This aligns with the similar observations by Mollah et al. ${ }^{15}$ A negative correlation between mean serum zinc level and the temperature was observed by Okposio et al. The reason for this inverse relationship is still speculative and the observation has not been replicated in the current study.

The exact pathogenesis of reduced zinc levels and febrile seizures susceptibility remains conjectural. Experimental synaptic zinc transporter knocked out mouse model, demonstrating an increased vulnerability to heat-induced seizures. ${ }^{6}$ This enhanced susceptibility to febrile seizures is hypothesized to lower synaptic concentrations of zinc and its poor constraint of excitatory output modulated by NMDA receptors. This unconstrained NMDA excitability secondary to diminished synaptic zinc concentrates is one of the proposed mechanisms. The enzyme modulatory function of zinc on pyridoxal kinase and resultant GABA synthesis have also been suggested. The full complexity of the role of zinc in epileptogenesis is still unestablished and merits attention.

In conclusion, the present study attends to the existing literature on relatively lower serum zinc concentrations in children with febrile seizures. Hypozincemia in one-third of the study population deserves special mention. The possible role of hypozincemia in febrile seizures, when compared with fever-matched controls without convulsions, needs to be addressed. A combination of zinc concentrates in plasma andCSF with zinc transporter gene polymorphisms may not only shed light on the proposed etiopathogenesis but alsooffer treatment opportunities. 


\section{Ethical Approval}

An informed consent form was signed by the parents of the patient to approve the use of patient information or material for scientific purposes. The patient identity has not been disclosed anywhere in the manuscript and does not contain any identifiable images.

\section{Funding}

None.

Conflict of Interest

None declared.

\section{References}

1 Gupta A. Febrile Seizures. Continuum (Minneap Minn) 2016;22(1 Epilepsy) :51-59

2 Steering Committee on Quality Improvement and Management, Subcommittee on Febrile Seizures American Academy of Pediatrics. Febrile seizures: clinical practice guideline for the long-term management of the child with simple febrile seizures. Pediatrics 2008;121(6):1281-1286

3 Capovilla G, Mastrangelo M, Romeo A, Vigevano F. Recommendations for the management of "febrile seizures" Ad hoc Task Force of LICE Guidelines Commission. Epilepsia 2009;50(Suppl 1) :2-6

4 Deng H, Zheng W, Song Z. The genetics and molecular biology of fever-associated seizures or epilepsy. Expert Rev Mol Med 2018;20:e3

5 Saghazadeh A, Mahmoudi M, Meysamie A, Gharedaghi M, Zamponi GW, Rezaei N. Possible role of trace elements in epilepsy and febrile seizures: a meta-analysis. Nutr Rev 2015;73(11):760-779
6 Hildebrand MS, Phillips AM, Mullen SA, et al. Loss of synaptic $\mathrm{Zn2+}$ transporter function increases risk of febrile seizures. Sci Rep 2015;5:17816

7 Okposio M, Sadoh W, Ofovwe G, Onyiriuka A. Serum zinc level in Nigerian children with febrile convulsion. J Pediatr Neurol 2015;10(3):187-191

8 Nasehi MM, Sakhaei R, Moosazadeh M, Aliramzany M. Comparison of serum zinc levels among children with simple febrile seizure and control group: a systematic review. Iran J Child Neurol 2015;9(1):17-24

9 Çelik K, Çelik Güzel E, Nalbantoğlu B, et al. Serum zinc levels in febrile seizures: is deficiency a risk factor? Turkiye Klinikleri J Pediatr 2012;21(1):1-6

10 Abdel Hameed ZA, El-Tellawy MM, Embaby M, Kamel YS. Relation of iron and zinc deficiencies to the occurrence of febrile convulsions. J Pediatr Neurosci 2019;14(2):61-64

11 Hosseini F, Nikkhah A, Afkhami Goli M. Serum zinc level in children with febrile seizure. Iran J Child Neurol 2020;14(1):43-47

12 Wojciak RW, Mojs E, Stanislawska-Kubiak M, Samborski W. The serum zinc, copper, iron, and chromium concentrations in epileptic children. Epilepsy Res 2013;104(1-2):40-44

13 Roohani N, Hurrell R, Kelishadi R, Schulin R. Zinc and its importance for human health: an integrative review. J Res Med Sci 2013;18(2):144-157

14 Lee J-H, Kim JH. Comparison of serum zinc levels measured by inductively coupled plasma mass spectrometry in preschool children with febrile and afebrile seizures. Ann Lab Med 2012;32(3):190-193

15 Mollah MAH, Rakshit SC, Anwar KS, et al. Zinc concentration in serum and cerebrospinal fluid simultaneously decrease in children with febrile seizure: findings from a prospective study in Bangladesh. Acta Paediatr 2008;97(12):1707-1711 